\title{
Statistics of Impedance, Local Density of States, and Reflection in Quantum Chaotic Systems with Absorption.
}

\author{
Yan V. Fyodorov ${ }^{1}$ and Dmitry V. Savin ${ }^{2}$ \\ ${ }^{1}$ Department of Mathematical Sciences, Brunel University, Uxbridge UB83PH, United Kingdom \\ ${ }^{2}$ Fachbereich Physik, Universität Duisburg-Essen, 45117 Essen, Germany
}

(Dated: September 6, 2004)

\begin{abstract}
We are interested in finding the joint distribution function of the real and imaginary parts of the local Green function for a system with chaotic internal wave scattering and a uniform energy loss (absorption). For a microwave cavity attached to a single-mode antenna the same quantity has a meaning of the complex cavity impedance. Using the random matrix approach, we relate its statistics to that of the reflection coefficient and scattering phase and provide exact distributions for systems with $\beta=2$ and $\beta=4$ symmetry class. In the case of $\beta=1$ we provide an interpolation formula which incorporates all known limiting cases and fits excellently available experimental data as well as diverse numeric tests.

PACS numbers: 05.45.Mt, 42.25.Bs, 73.23.-b
\end{abstract}

Characterising statistical fluctuations of physical observables in quantum systems with underlying chaotic classical dynamics remains a very active field of research in theoretical and experimental physics. A considerable progress in understanding the phenomenon was underpinned by revealing the apparent universality of the fluctuations in systems of very diverse microscopic nature, ranging from atomic nuclei and Rydberg atoms in strong external fields, to complex molecules, quantum dots, and mesoscopic samples, see e.g. [1]. From theoretical side, the universality allows one to exploit the random matrix theory (RMT) as a powerful tool for analysis of generic features of the energy spectra of such systems [2, 3].

In many atomic, molecular, and mesoscopic systems the quantity which is readily obtained experimentally is the absorption spectrum for transitions from a given initial state $|g\rangle$ to highly excited chaotic states at the energy $E$. For high-resolution experiments chaotic spectra consists of wellresolved narrow resonance peaks and one can, in principle, study statistics of the peak heights and widths as well as that of spacings between consecutive peaks. Most frequently, however, the absorption spectra look practically continuous due to both inevitable level broadening and finite experimental resolution. Then the relevant statistics is the distribution and correlation functions of the absorption probability $\sigma(E)$ (also known as the strength function of the dipole operator $\hat{\mu}$ ) which in the simplest situation of uniform level broadening $\Gamma$ is given by $\sigma(E) \propto \operatorname{Im}\langle g|\hat{\mu} \hat{G}(E) \hat{\mu}| g\rangle$, see e.g. Ref. [4] and discussion therein. Assuming the validity of the RMT, the problem then amounts to studying statistical properties of the resolvent (Green function) operator $\hat{G}(E) \equiv(E+i \Gamma / 2-\hat{H})^{-1}$ associated with the random matrix $\hat{H}$ which replaces the actual chaotic Hamiltonian. In particular, the imaginary part of the diagonal entries of $\hat{G}(E)$ is well known in the solid state physics as the local density of states (LDoS) and in this capacity its statistics enjoyed many studies [4, 5, 6, 7, 8].

From the experimental point of view the same universality, which makes the use of the RMT legitimate, provides one with an attractive possibility to employ simple model systems for analyzing generic statistics of the fluctuating quantities. One of such systems which proved to be an ideal playground for investigating a variety of quantum chaos phenomena are various microwave billiards [1]. The billiards are realized as resonators in a form of electromagnetic cavities fed up with the radiation of the wavelength small compared with the characteristic size of the enclosure and shaped to ensure the chaoticity of internal scattering. The cavities are coupled to transmission lines or to waveguides which are used to inject electromagnetic waves into the system as well as to collect the outgoing waves. The adequate description of the experiment is then achieved in terms of the scattering matrix $S$ relating amplitudes of incoming and outgoing waves.

High-resolution experiments are usually performed in lowtemperature (superconducting) cavities with very high quality factor [9]. Majority of the experiments are, however, done at room temperatures [10, 11, 12, 13]. Inevitable energy losses (absorption) leading to uniform broadening of the resonances play, therefore, an important role and have to be taken into account adequately when describing the experiments theoretically.

In the present paper we are going to concentrate on the simplest case of a single one-channel antenna experiment. To be able to employ the RMT methods, it is conventional to represent the resonant part of the scattering matrix $S$ in the following form (see e.g. Ref. [14]):

$$
S(E)=\frac{1-i K(E)}{1+i K(E)} \equiv \sqrt{r} e^{i \theta}
$$

where $K(E)=V^{\dagger} \hat{G}(E) V$ is the so-called $K$ matrix. The Hamiltonian $\hat{H}$ of the closed chaotic cavity gives rise to $N$ eigenfrequencies $E_{n}$ characterized in the relevant range of the scattering energy $E$ by the mean level spacing $\Delta$. The column-vector $V$ describes the energy-independent amplitudes coupling the corresponding eigenmodes to the propagating mode in the antenna. We see again that the study of statistical properties of the scattering matrix amounts to knowing statistics of the diagonal element $G_{11}$ of the Green function of the closed system in some basis. In fact, in the present context the function $i K \equiv Z$ has the direct physical meaning of 
the electric impedance $Z$ of the cavity which relates linearly voltages and currents at the antenna port [11].

Without absorption $\Gamma=0$ and the scattering matrix is unimodular: $r \equiv 1$. At finite absorption, $\Gamma>0$, the reflection coefficient $r$ and the scattering phase $\theta$ have nontrivial distributions, which have been recently measured in experiment [10, 13]. On the other hand, universal fluctuations of both real and imaginary parts of the cavity impedance $Z$ have been recently investigated experimentally in [11]. Since the impedance matrix $Z$ is related to eigenmodes and eigenfrequencies of the closed cavity, the study of $Z$ is in some sense complementary to that of $S$.

Various statistics related to the scattering matrix of chaotic systems with losses were subject of a number of recent papers [15, 16, 17, 18, 19, 20]. Explicit analytical results were available, however, only for the simplest case of systems with no time-reversal invariance (TRI) corresponding to the so-called $\beta=2$ symmetry class of the RMT. At the same time, majority of the billiard-type experiments is performed in systems which are time-reversal invariant $(\beta=1$ symmetry class of the RMT). Similar situation holds for the statistics of the local Green function, in particular, LDoS. For the $\beta=2$ case the corresponding expressions were obtained by various methods in [5, 6, 7, 21]. An attempt [8] to provide an expression for the LDoS distribution for the $\beta=1$ case can not be considered as particularly successful, as the general expression was given in a form of an intractable fivefold integral. Finally, it is worth mentioning the existence of the $\beta=4$ symmetry class describing time-reversal invariant chaotic systems with half-integer spin. This situation may occur in quantum dots with strong spin-orbit scattering [22], in Rydberg atoms driven by microwave fields [23], and can be efficiently simulated in some other models of quantum chaotic systems [24].

The fundamental quantity which determines the full statistics of $S$ or $Z$ is the joint distribution function $\mathcal{P}(u, v)$ of the real $u=\operatorname{Re} K$ and imaginary $v=-\operatorname{Im} K>0$ parts of $K$. Generally, one can always write $K=\kappa(N \Delta / \pi) G_{11}$ in the RMT. The effective coupling constant $\kappa=\pi\|V\|^{2} / N \Delta>0$ enters the $S$ matrix statistics only through the so-called transmission coefficient $T \equiv 1-|\bar{S}|^{2}\left(=4 \kappa /(1+\kappa)^{2}\right.$ in the middle of the spectrum, $E=0$ ), see e.g. Ref. [14] for details.

A convenient starting point of our analysis is the observation that the distribution $\mathcal{P}(u, v)$ must always have the following general form:

$$
\mathcal{P}(u, v)=\frac{1}{2 \pi v^{2}} P_{0}(x),
$$

with $x=\left(u^{2}+v^{2}+1\right) / 2 v>1$. It initially emerged in [25] in the course of explicit calculations for the $\beta=2$ symmetry class, but neither origin nor generality of such a form were appreciated. Here we show that Eq. (2) is the direct consequence of two fundamental properties of the so-called "perfect coupling" case $T=1$ : (i) the statistical independence of the $S$ matrix modulus $r \equiv \frac{x-1}{x+1}$ and its phase $\theta$; and (ii) the uniform distribution of $\theta \in(0,2 \pi)$. Both these properties can be verified using the methods of Ref. [26]. The joint distri- bution $P(x, \theta)$ then factorizes to $P_{0}(x) / 2 \pi$. Choose now new variable $y \equiv \frac{\operatorname{Re} S}{\operatorname{Im} S}=\cot (\theta)$ instead of $\theta$, so that $|d \theta / d y|=$ $\left(1+y^{2}\right)^{-1}$. Noticing that $y=\left(u^{2}+v^{2}-1\right) / 2 u$ and evaluating the corresponding Jacobian $|\partial(x, y) / \partial(u, v)|=\left(1+y^{2}\right) / v^{2}$ of the transformation from $x$ and $y$ to $u$ and $v$, we come after a simple calculation to (2).

The explicit form of $P_{0}(x)$ at arbitrary absorption for various symmetry classes will be given and discussed below. Having $P_{0}(x)$ at our disposal, it is immediate to find the distribution of the imaginary part $v$ (the LDoS normalized for convenience to have the unit mean value):

$$
\mathcal{P}_{v}(v)=\frac{\sqrt{2}}{\pi v^{3 / 2}} \int_{0}^{\infty} d q P_{0}\left[q^{2}+\frac{1}{2}\left(v+\frac{1}{v}\right)\right],
$$

The distribution is normalized to 1 and has the first moment unity, $\langle v\rangle \equiv \int_{0}^{\infty} d v v \mathcal{P}(v)=1$, automatically due to invariance of the integrand with respect to the change $v \rightarrow 1 / v$. Similarly, one can find the distribution of the real part $u$ to be:

$$
\mathcal{P}_{u}(u)=\frac{1}{2 \pi \sqrt{u^{2}+1}} \int_{0}^{\infty} d q P_{0}\left[\frac{\sqrt{u^{2}+1}}{2}\left(q+\frac{1}{q}\right)\right] .
$$

Although $u$ has no direct physical meaning in the context of solid state mesoscopic systems, both $\mathcal{P}_{u}(u)$ and $\mathcal{P}_{v}(v)$ are directly measurable in microwave cavities [11].

Let us now discuss the explicit forms of $P_{0}(x)$ for various symmetry classes: $\beta=1,2,4$. For the simplest case of broken TRI $(\beta=2)$ an exact result is available at arbitrary values of the dimensionless absorption strength $\gamma \equiv 2 \pi \Gamma / \Delta[17,18,19]$. Scaling the absorption parameter for the subsequent use as $\alpha \equiv \gamma \beta / 2$, we can represent the $\beta=2$ result as follows:

$$
P_{0}(x)=\frac{\mathcal{N}_{\beta}}{2}\left[A(\alpha(x+1) / 2)^{\beta / 2}+B\right] e^{-\alpha(x+1) / 2},
$$

with $\alpha$-dependent constants $A \equiv e^{\alpha}-1$ and $B \equiv 1+\alpha-e^{\alpha}$ and the normalization constant $\mathcal{N}_{2}=1$.

For the case $\beta=4$ the exact form became available very recently [31] by exploiting important advances in the RMT [32]. The explicit derivation will be given elsewhere, the final result being [31]:

$$
P_{0}^{\text {gse }}(x)=\widetilde{P}_{0}^{\text {gue }}(x)+C(x, \gamma) e^{-\gamma x} \int_{0}^{\gamma} d t \frac{\sinh t}{t},
$$

where $\widetilde{P}_{0}^{\text {gue }}(x)$ is the distribution (5) for $\beta=2$ taken, however, at $\alpha=2 \gamma$ and $C(x, \gamma) \equiv \gamma^{2}(x+1)^{2} / 2-\gamma(\gamma+1)(x+1)+\gamma$.

Unfortunately, for the most interesting case $\beta=1$ the explicit formula for $P_{0}(x)$ is not available yet, apart from the limiting cases of weak or strong absorption:

$$
P_{0}(x) \simeq \begin{cases}\frac{\alpha^{\beta / 2+1}}{2 \Gamma(\beta / 2+1)}\left(\frac{x+1}{2}\right)^{\beta / 2} e^{-\alpha(x+1) / 2}, & \gamma \ll 1 \\ \alpha e^{-\alpha(x-1) / 2}, & \gamma \gg 1 .\end{cases}
$$

The first line here results from the relation $\frac{2}{x+1}=1-r \approx \gamma \tau$ at $\gamma \ll 1$ between the reflection coefficient and the (dimensionless) time-delay $\tau$, see [15, 17]. The time-delay distributions are known [14, 27] for all $\beta=1,2,4: \mathcal{P}_{\tau}(\tau)=$ 

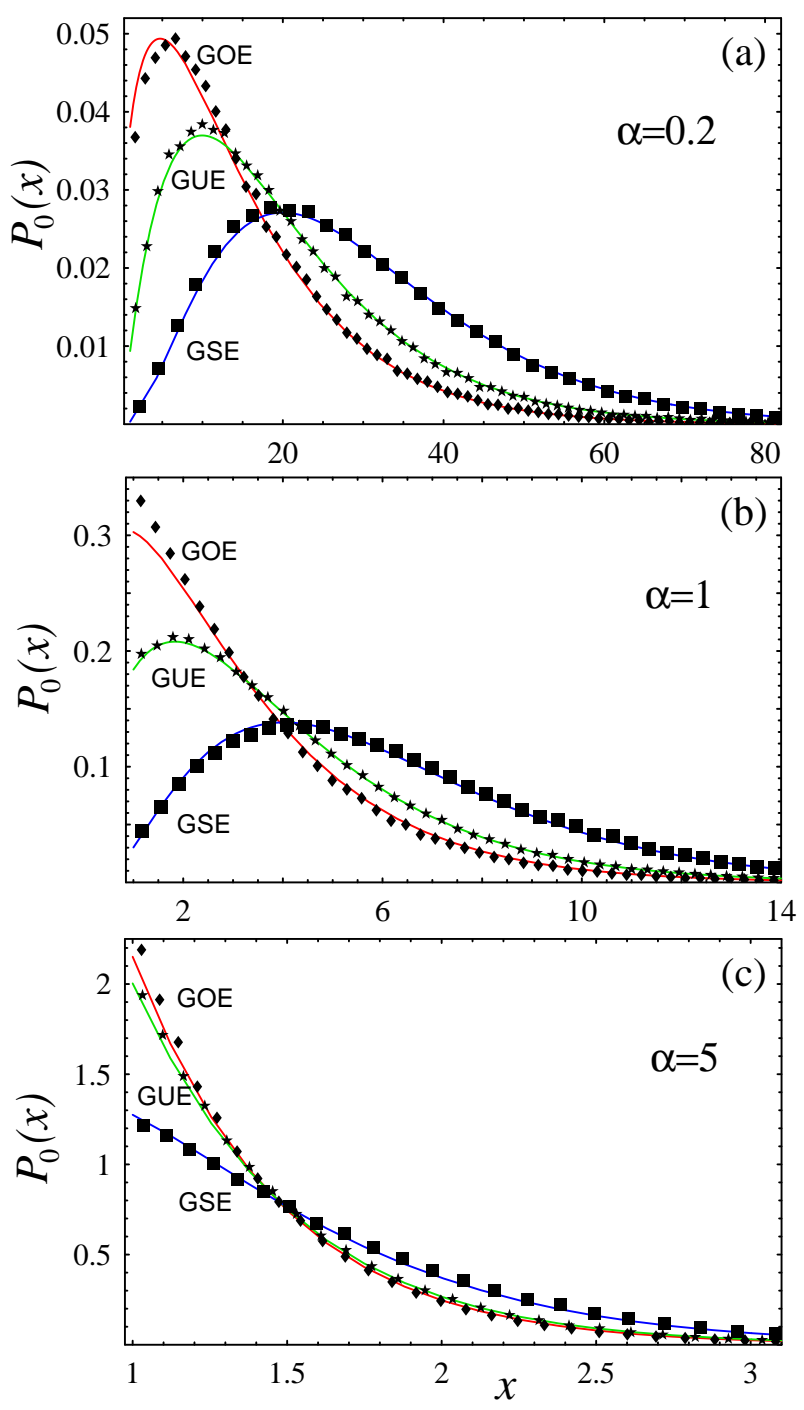

FIG. 1: The distribution $P_{0}(x)$, Eq. (5), at different values of the absorption parameter $\alpha=\beta \pi \Gamma / \Delta$. Solid lines correspond to the $\beta=1$ (GOE) and 2 (GUE) cases and to the exact $\beta=4$ (GSE) result, Eq. 6. Symbols stand for the numerics done for 400 realizations of $500 \times 500$ random matrices.

$\left[(\beta / 2)^{\beta / 2} / \Gamma(\beta / 2)\right] \tau^{-\beta / 2-2} e^{-\beta / 2 \tau}$. In the opposite case $\gamma \gg$ 1, the known limiting Rayleigh distribution [16] $P(r) \simeq$ $(\gamma \beta / 2) e^{-r \gamma \beta / 2}$ yields the second line in Eq.(7)

In the absence of the general expression for $\beta=1$ a natural idea is to try to invent a formula interpolating between the known limiting cases [13]. We suggest Eq. (5) to be the appropriate natural candidate, with the normalization constant being $\mathcal{N}_{\beta}=\alpha /\left(A \Gamma(\beta / 2+1, \alpha)+B e^{-\alpha}\right)$, where $\Gamma(\nu, \alpha)=$ $\int_{\alpha}^{\infty} d t t^{\nu-1} e^{-t}$. Indeed, such a form reproduces correctly Eq. (7) as both limits are determined solely by the first (universal) term in (5). One needs, however, to keep $B$ in order to handle properly the case of moderate absorption $(\alpha \sim 1)$.

Figure 1 shows results of numerical simulations with random matrices drawn from the Gaussian orthogonal (GOE, $\beta=1$ ), unitary (GUE, $\beta=2$ ) or symplectic (GSE, $\beta=4$ ) en- semble. The overall agreement of Eq. (5) with the data at $\beta=1$ is nearly as good as for the exact cases $\beta=2,4$. Another check has been performed in Ref. [13] which measured distributions of the reflection coefficient and the scattering phase in the broad range of system parameters and found very good agreement with the corresponding expressions following from Eq. (5).

We discuss now the behavior of $\mathcal{P}_{v}(v)$ and $\mathcal{P}_{u}(u)$. Performing the integration in Eq. (3), we arrive for $\beta=2$ at

$$
\begin{aligned}
\mathcal{P}_{v}^{\text {gue }}(v)= & (\gamma / 16 \pi)^{1 / 2} v^{-3 / 2} \exp \left[-\gamma\left(v+v^{-1}\right) / 4\right] \\
& \times\left[2 \cosh \frac{\gamma}{2}+\left(v+v^{-1}-2 / \gamma\right) \sinh \frac{\gamma}{2}\right],
\end{aligned}
$$

which is exactly the LDoS distribution obtained earlier in [5]. For the case $\beta=1$, we get the following result:

$$
\mathcal{P}_{v}^{\mathrm{goe}}(v)=\frac{\mathcal{N}_{1} e^{-a}}{\pi \sqrt{2 \gamma} v^{3 / 2}}\left(A\left[K_{0}(a)+K_{1}(a)\right] a+\sqrt{\pi} B e^{-a}\right),
$$

with $a \equiv \frac{\gamma}{16}(\sqrt{v}+1 / \sqrt{v})^{2}$ and $K_{\nu}(z)$ being the MacDonald function. It is instructive to consider the asymptotic behavior of these functions in the limits of small or large absorption. At $\gamma \ll 1$, the distribution $\mathcal{P}_{v}(v)$ becomes very broad having the maximum at $v \sim \gamma$, a power-law bulk behavior and an exponential cutoff at the far tails:

$$
\mathcal{P}_{v}(v) \propto\left\{\begin{array}{l}
\alpha^{(1+\beta) / 2} v^{-(3+\beta) / 2} e^{-\alpha / 4 v}, \quad v \ll \alpha \\
\alpha^{1 / 2} v^{-3 / 2}, \quad \alpha \ll v \ll 1 / \alpha \\
\alpha^{(1+\beta) / 2} v^{-(3-\beta) / 2} e^{-\alpha v / 4}, \quad 1 / \alpha \ll v,
\end{array}\right.
$$

where constants $\sim 1$ are omitted. This result can be physically interpreted in the single-level approximation [5, 8], when the bulk and tail behavior is governed by spectral and wave function fluctuations, respectively. As $\gamma$ increases, a number of levels contributing to $v$ grows as $\sim \gamma$, so that $\mathcal{P}_{v}(v)$ tends to the limiting Gaussian distribution

$$
\mathcal{P}_{v}(v)=\sqrt{\frac{\alpha}{4 \pi v^{3}}} \exp \left[-\frac{\alpha}{4}\left(\sqrt{v}-\frac{1}{\sqrt{v}}\right)^{2}\right]
$$

which has a peak at $v \sim 1$ of the width $\propto 1 / \sqrt{\gamma} \ll 1$, in agreement with the earlier result [8].

As to the distribution $\mathcal{P}_{u}(u)$, equation (4) leads after the integration to the following exact $\beta=2$ result:

$$
\mathcal{P}_{u}^{\text {gue }}(u)=\frac{\gamma}{2 \pi}\left[\sinh \frac{\gamma}{2} K_{0}\left(\frac{\gamma \tilde{u}}{2}\right)+\frac{\cosh \frac{\gamma}{2}}{\tilde{u}} K_{1}\left(\frac{\gamma \tilde{u}}{2}\right)\right],
$$

where $\tilde{u} \equiv \sqrt{u^{2}+1}$. Integrating the interpolation formula for the case $\beta=1$, we obtain

$$
\mathcal{P}_{u}^{\mathrm{goe}}(u)=\frac{\mathcal{N}_{1} e^{-\gamma / 4}}{2 \pi \tilde{u}}\left[\frac{A}{2} \sqrt{\frac{\gamma}{4}} D\left(\frac{\tilde{u}}{2}\right)+B K_{1}\left(\frac{\gamma \tilde{u}}{4}\right)\right],
$$

where $D(z) \equiv \int_{0}^{\infty} d q \sqrt{1+z\left(q+q^{-1}\right)} e^{-\gamma z\left(q+q^{-1}\right) / 4}$ is introduced for convenience. The limiting forms of $\mathcal{P}_{u}(u)$ at weak 
and strong absorption follow readily as

$$
\mathcal{P}_{u}(u) \simeq \begin{cases}\pi^{-1}\left(1+u^{2}\right)^{-1}, & \alpha \ll 1 \\ \sqrt{\alpha / 4 \pi} e^{-\alpha u^{2} / 4}, & \alpha \gg 1,\end{cases}
$$

and describe a crossover from the Lorentzian to Gaussian distribution as absorption grows. This type of behavior as well as the trend of $\mathcal{P}_{v}(v)$ to the Gaussian (11) was recently observed in the experimental study of the cavity impedance [11].

Finally, let us mention that the case of nonperfect coupling, $T<1$, can be mapped [28, 29] onto that of perfect one making use of the parametrization [30] $S_{0}=(S-\sqrt{1-T}) /(1-$ $\sqrt{1-T} S)$. Here $S_{0}$ is the scattering matrix of the system in the perfect coupling case. Now $x$ and $\theta$ do correlate and, after the evaluation in parametrization (1) of the corresponding Jacobian, the joint distribution $P(x, \theta)$ is found to be

$$
P(x, \theta)=\frac{1}{2 \pi} P_{0}\left(x g-\sqrt{\left(x^{2}-1\right)\left(g^{2}-1\right)} \cos \theta\right),
$$

with $g \equiv 2 / T-1$. Complementary to Eq. (2), equation (15) provides an access to scattering observables. The integration there over $x$ yields the scattering phase distribution. In particular, when absorption vanishes, $x \rightarrow \infty(r \rightarrow 1)$ and $P_{0}(x) \rightarrow \delta(1 / x)$, giving readily $P(x, \theta)=\rho(\theta) \delta(1 / x)$, with the phase density $\rho(\theta)=\left[2 \pi\left(g-\sqrt{g^{2}-1} \cos \theta\right)\right]^{-1}$ found earlier [29]. As another example, the distribution of the reflection coefficient in terms of $P_{0}(x)$ is given at arbitrary coupling by (cf. Eq.(5) in [19] and see also [13, 18] in this respect):

$$
P_{r}(r)=\int_{0}^{2 \pi} \frac{d \theta \pi^{-1}}{(1-r)^{2}} P_{0}\left[\frac{2\left(g-\sqrt{g^{2}-1} \sqrt{r} \cos \theta\right)}{1-r}-g\right] .
$$

In conclusion, although rigorous analytical treatment of the $\beta=1$ case remains a theoretical challenge it is worth stressing, however, that the suggested interpolation formulas should be sufficient for the most of practical purposes of comparison to the experimental/numerical data. Moreover, all physically interesting limiting cases, as e.g. Eqs. (10), 11) and (14), are already reproduced from the exact limiting statistics (7). At the same time, it is important to understand that an excellent performance of the interpolation formula (5) for $\beta=1$ is nothing else as a lucky coincidence. Indeed, applying the same formula for the $\beta=4$ case, we have found that apart from well reproduced limits of weak and strong absorption, an agreement with numerics at intermediate values $\gamma \sim 1$ turns out to be by far not as good as in the GOE case.

We are grateful to S. Anlage, P. Brouwer, U. Kuhl, A. Mirlin, H.-J. Sommers and E. Strahov for useful communications at various stages of this work. The financial support by the SFB/TR 12 der DFG is acknowledged with thanks.
[1] H.-J. Stöckmann, Quantum Chaos: An Introduction (Cambridge University Press, Cambridge,1999).

[2] T. Guhr, A. Müller-Groeling, and H. A. Weidenmüller, Phys. Rep. 299, 189 (1998).

[3] Y. Alhassid, Rev. Mod. Phys. 72, 895 (2000).

[4] Y. V. Fyodorov and Y. Alhassid, Phys. Rev. A 58, R3375 (1998).

[5] K. B. Efetov and V. N. Prigodin, Phys. Rev. Lett. 70, 1315 (1993).

[6] C. W. J. Beenakker, Phys. Rev. B 50, 15170 (1994).

[7] A. D. Mirlin and Y. V. Fyodorov, Europhys. Lett. 25, 669 (1994).

[8] N. Taniguchi and V. N. Prigodin, Phys. Rev. B 54, 14305 (1996).

[9] C. Dembowski et al., Phys. Rev. Lett. 89, 064101 (2002).

[10] R. A. Méndez-Sánchez et al., Phys. Rev. Lett. 91, 174102 (2003).

[11] S. Hemmady et al., e-print cond-mat/0403225, X. Zheng et al., e-prints cond-mat/0408317 and cond-mat/0408327

[12] J. Barthélemy, O. Legrand, and F. Mortessagne, e-prints cond-mat/0401638 and cond-mat/0402029

[13] U. Kuhl et al., e-print cond-mat/0407197

[14] Y. V. Fyodorov and H.-J. Sommers, J. Math. Phys. 38, 1918 (1997).

[15] S. A. Ramakrishna and N. Kumar, Phys. Rev. B 61, 3163 (2000).

[16] E. Kogan, P. A. Mello, and H. Liqun, Phys. Rev. E 61, R17 (2000).

[17] C. W. J. Beenakker and P. W. Brouwer, Physica E 9, 463 (2001).

[18] D. V. Savin and H.-J. Sommers, Phys. Rev. E 68, 036211 (2003).

[19] Y. V. Fyodorov, JETP Lett. 78, 250 (2003).

[20] I. Rozhkov et al., Phys. Rev. E 68, 016204 (2003); Y. V. Fyodorov and A. Ossipov, Phys. Rev. Lett. 92, 084103 (2004).

[21] A. V. Andreev and B. D. Simons, Phys. Rev. Lett. 75, 2304 (1995).

[22] I. L. Aleiner and V. I. Fal'ko, Phys. Rev. Lett. 87, 256801 (2001).

[23] K. Sacha and J. Zakrzewski, Phys. Rev. Lett. 86, 2269 (2001).

[24] J. Bolte and J. Harrison, J. Phys. A 36, 2747 (2003).

[25] A. D. Mirlin and Y. V. Fyodorov, Phys. Rev. Lett. 72, 526 (1994); J. Phys. I 4, 655 (1994).

[26] P. W. Brouwer and C. W. J. Beenakker, Phys. Rev. B 55, 4695 (1997).

[27] V. A. Gopar, P. A. Mello, and M. Büttiker, Phys. Rev. Lett. 77, 3005 (1996).

[28] P. W. Brouwer, Phys. Rev. B 51, 16878 (1995).

[29] D. V. Savin, Y. V. Fyodorov, and H.-J. Sommers, Phys. Rev. E 63, 035202(R) (2001).

[30] P. A. Mello, P. Pereyra, and T. Seligman, Ann. Phys. 161, 254 (1985).

[31] Y. V. Fyodorov, unpublished.

[32] A. Borodin and E. Strahov, e-print math-ph/0407065 